\title{
An Electron Microscopy Study on Morphology and Microstructure of a NiCoAlFeMoTiCr High-entropy Alloy synthesized by Arc-melting
}

\author{
F.J. Baldenebro-Lopez ${ }^{1,2}$, C.D. Gómez-Esparza ${ }^{1}$, J.A. Baldenebro-Lopez ${ }^{2}$, I. Estrada-Guel ${ }^{3}$, J.T. \\ Elizalde-Galindo ${ }^{1}$, C.A. Rodríguez-González ${ }^{1}$, R. Martínez-Sánchez ${ }^{3}$ and H. Camacho-Montes ${ }^{1, *}$ \\ 1. IIT-Universidad Autónoma de Ciudad Juárez., 32310 Ciudad Juárez, Chih., México. \\ 2. FIM-Universidad Autónoma de Sinaloa, 81223 Los Mochis, Sinaloa, México. \\ 3. Centro de Investigación en Materiales Avanzados (CIMAV), 31109 Chihuahua, Chih., México.
}

High-entropy alloys (HEAs) contain at least five principal elements with concentrations between 5 and $35 \%$ and have received large attention from the materials science community since they were introduced about a decade ago by J.W. Yeh [1] this due to their extraordinary properties such as high hardness, high corrosion resistance and excellent high temperature resistance [2]. This new class of alloys is, in principle, expected to exhibit a large degree of mutual solubility in a single-phase, instead of complex ordered intermetallics. An example of such a single-phase HEA is the equiatomic CoCrFeMnNi alloy first reported by Cantor et al. [3], however, the majority of HEAs is actually multiphase with simple FCC and/or BCC structure.

In this work, elemental powders supplied by Alfa Aesar with purity higher than $99.5 \%$ in weight $\mathrm{Ni}$, Co, $\mathrm{Al}, \mathrm{Fe}, \mathrm{Mo}, \mathrm{Ti}$ and $\mathrm{Cr}$ were initially weighed and mixed. The MA was performed in a high-energy ball mill (SPEX-8000M) for $10 \mathrm{~h}$ periods, under an argon atmosphere. Methanol was used as a process control agent to avid metal agglomeration. The milled powders were mechanically pressed into cylindrical samples with $10 \mathrm{~mm}$ of diameter under $1.5 \mathrm{GPa}$ of pressure, using a uniaxial compaction die in a hydraulic press and subsequent by melting arc under an argon atmosphere to prevent oxidation. The ingots were remelted at least 5 times to improve chemical homogeneity. The morphological and microstructural features were characterized by scanning electron microscopy (SEM, JEOL JSM-7401F) and phase composition was identified by transmission electron microscopy (TEM, JEOL-JEM2200FS).

After alloying process, a homogeneous morphology can be achieved with the continuous competition between plastic deformation, agglomeration and size reduction. At the same time, the chemical composition of individual MA'ed powder is very similar to the proportion of the starting elemental powders, which was corroborated based on EDS-SEM analyses (See Fig. 1); although this technique is limited to being semi-quantitative, it was used to confirm that the chemical composition of the alloys was nearly equiatomic. It is possible to assume a good chemical homogeneity of the powder alloys with a variation of $\pm 3 \%$. In order to corroborate if the crystal size of the obtained phases was in the nanoscale, the powders were also investigated by TEM analyses. Fig. 2 shows typical dark field images and the corresponding SAD pattern of the mechanically alloyed powders for $10 \mathrm{~h}$. It can be noticed that crystal average size of powders after $10 \mathrm{~h}$ of milling is $30 \mathrm{~nm}$, approximately. Fig. 3 shows SEM images of a representative microstructure after arc melting of the as-cast samples from milled powders. The sample exhibit the formation of three phases, a gray phase (Ni-Ti-Co), a continuous bright phase (Ni-Ti) and discontinuous bright phase (Fe-Cr). Z-contrast TEM image of the as-cast sample of Fig. 4 shows the presence of same phases detected in the Fig. 3, which was determined by EDS/TEM analysis.

An equiatomic NiCoAlFeMoTiCr high-entropy alloys from alloyed powders were produced under an argon atmosphere by arc melting. Nanometric crystallite size was observed in the alloyed powders. The 
EDS analysis shows the presence of all elements in the three phases present in the microstructure, providing evidence that exist concentration of different elements according to present phases.

References:

[1] J.W. Yeh, et al., Adv. Eng. Mater. 6 (2004), p.299-303.

[2] M.H. Tsai, J.W. Yeh, Mater. Res. Lett. 2 (2014), p.107-123.

[3] B. Cantor, et al., Mater. Sci. Eng. A 375-377 (2004), p.213-218.

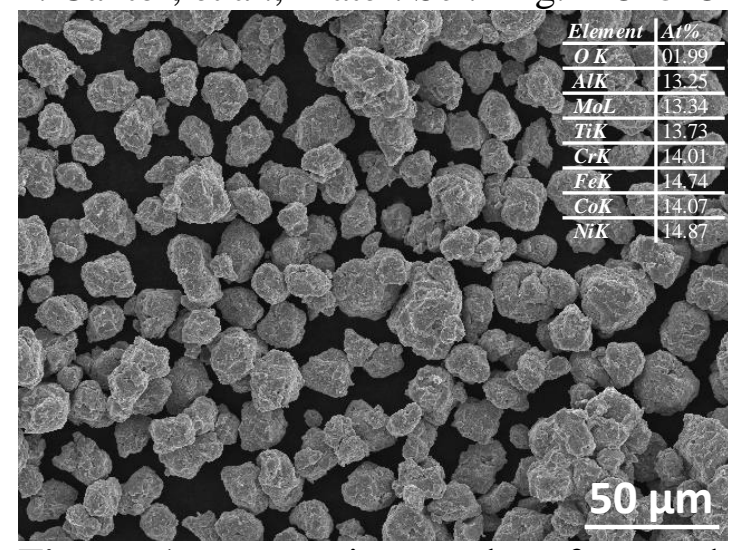

Figure 1. SEM micrographs of MA'ed NiCoAlFeMoTiCr powder alloy.

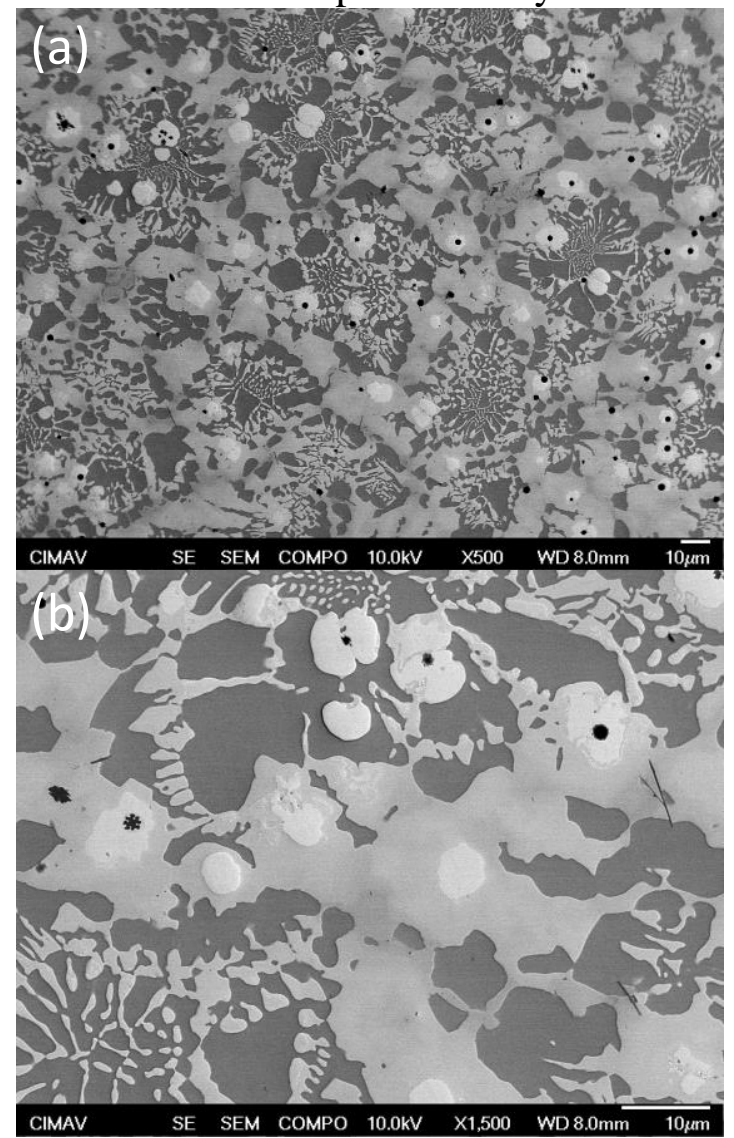

Figure 3. SEM images of the as-cast samples of NiCoAlFeMoTiCr milled powders: (a) 500X and (b) $1500 \mathrm{X}$.



Figure 2. TEM micrographs of NiCoAlFeMoTiCr powder alloy after $10 \mathrm{~h}$ of milling: (a) Dark field image and (b) SAED pattern.

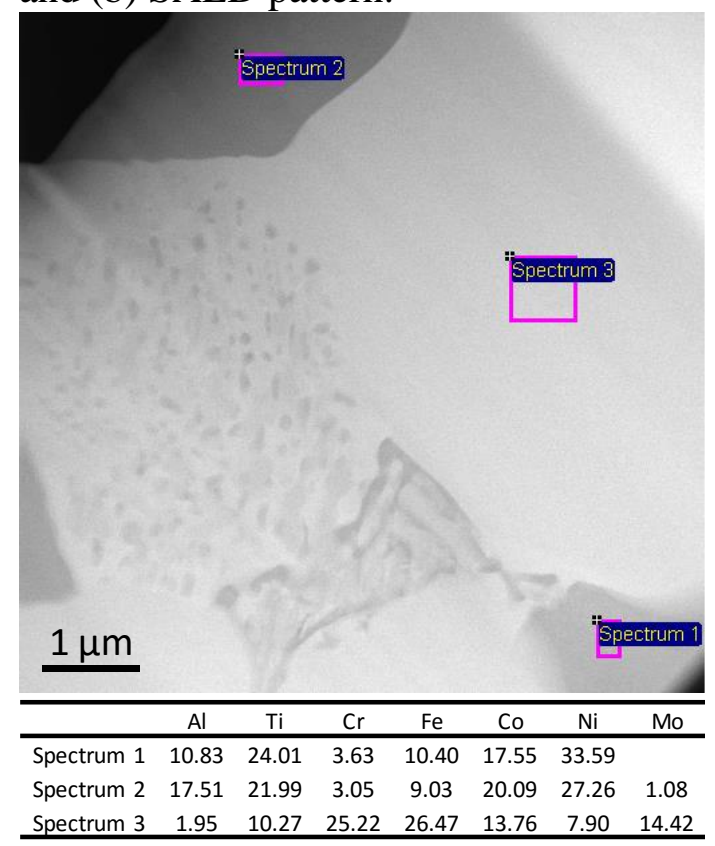

Figure 4. TEM image and EDS results showing the presence and chemical composition of the different phases present in the sample. 